\title{
Self-assembly of carbonate-silica colloids: between living and non-living form
}

\author{
Stephen T. Hyde ${ }^{a, *}$, Anna M. Carnerup ${ }^{\text {a }}$, \\ Ann-Kristin Larsson ${ }^{\mathrm{a}}$, Andrew G. Christy ${ }^{\mathrm{a}}$ and \\ Juan Manuel García-Ruiz ${ }^{b}$ \\ ${ }^{a}$ Department of Applied Mathematics, Research School of Physical Sciences and \\ Engineering, Australian National University, 0200 Canberra, ACT, Australia \\ ${ }^{\mathrm{b}}$ Laboratorio de Estudios Cristalograficos, Instituto Andaluz de Ciencias de la \\ Tierra, CSIC-Universidad de Granada, Ave. Fuentenueva s/n, 18002 Granada, \\ Spain
}

\begin{abstract}
We describe self-assembled silica-carbonate aggregates that show a diverse range of morphologies, all of which display complex internal structure, orientational ordering of components, and well-organised, curved global morphologies that bear a strong resemblance to biogenic forms. The internal order is described as a liquid-crystallike organisation of colloidal particles. We discuss possible causes for the striking morphologies of these inorganic materials, including local nanocrystal packing constraints and global silica membrane templating.
\end{abstract}

Key words: inorganic self-assembly, liquid crystals, silica-carbonate aggregates, biomineralisation

PACS: 81.16.Dn, 83.80.Xz, 61.66.Fn, 81.05.Zx, 82.70.Gg

\section{Introduction}

Self-assembly of inorganic colloids is an issue central to understanding of biomineralisation and related issues, such as recognition of fossils $[1,2]$. There are indications that inorganic colloids can form assemblies that mimic those of organic molecules in liquid crystals: smectic, cholesteric and nematic mesophases

* Corresponding author.

Email address: stephen.hyde@anu.edu.au (Stephen T. Hyde). 
have been reported $[3,4]$. Recently, complex smectic ordering has been reported for actin polymeric filaments [5], further blurring the distinction between mesostructure in hard and soft colloidal systems, and revealing a common lexicon of liquid-crystal-like mesostructure among biopolymers, inorganic colloids and organic molecules. Here a description of liquid crystal-like assemblies of composite inorganic carbonate-silica colloids is given. The local ordering mimics that found in chiral liquid crystals, though the physical details responsible for that ordering remain unknown. In addition, the external morphologies of these assemblies resemble in many aspects the forms previously believed to be the solely found in organic biological forms (curvilinear and non-facetted). We call our assemblies "biomorphs".

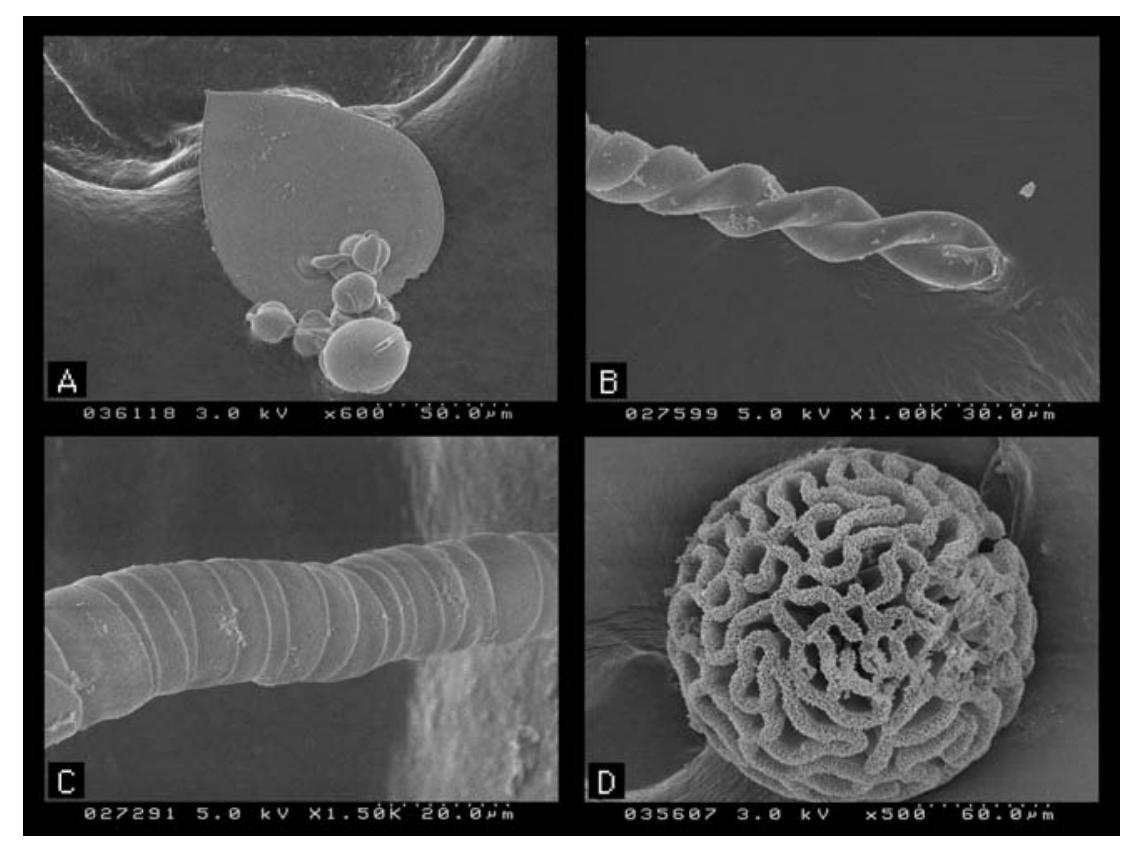

Fig. 1. FESEM images of a selection of various barium-carbonate biomorphs:A) sheet-like aggregate, B) a twisted ribbon, C) a tightly wound braid and D)a floral spherulite.

\section{Experimental growth of silica-carbonate biomorphs}

The materials are routinely obtained as precipitates in aqueous solution, at ambient temperature and pressure [6,7]. The preparation is related to that of standard colloidal precipitates. We focus here on precipitates of barium carbonate, though other alkaline earth salts also form related biomorphs. Alkaline water-glass solutions are prepared with distilled water and stock $\mathrm{Na}_{2} \mathrm{SiO}_{3}$ solutions (Sigma-Aldrich) The $\mathrm{pH}$ is adjusted with $0.1 \mathrm{M} \mathrm{NaOH}$ to give a value of around 11.3 followed by the addition of $\mathrm{BaCl}_{2}$ solution giving the final solution a silica concentration of about 400-900 ppm and a barium con- 
centration between $0.005-0.5 \mathrm{M}$. The diffusion of atmospheric $\mathrm{CO}_{2}$ into the solution leads to gradual formation of insoluble $\mathrm{BaCO}_{3}$. After a few hours microscopic particles are seen in an optical microscope, growing over a period of up to a day to aggregates visible to the naked eye. These silica-carbonate biomorphs are dramatically different in external form to carbonates precipitated in the absence of silica. Silica concentrations above about $250 \mathrm{ppm}$ (a sol) lead to characteristic biomorphic forms [7], while lower concentrations give well-known aggregate morphologies, ranging from colloidal fine-grained precipitates to larger crystals with twinned pseudo-rhombohedral morphology characteristic of the orthorhombic barium carbonate witherite structure. Biomorphs can grow in silica sols and more concentrated silica gels without marked differences in their forms [6].

In this paper we focus on the sheet-like, filamentous and flower-like spherulitic biomorphs (see Fig1). The sheets are typically cardoidal in form, with the original globular aggregate sometimes still visible at the cusp of the cardoid (see Fig1 A). The perimeter of the sheet is usually smoothly folded over, presenting a quasi-tubular rim. The rim often tapers to a point, from which a filament grows. Filaments can extend over very long lengths (microns in sols, $\mathrm{mm}$. in gels), though the distribution of their diameters is strictly bimodal, with finer braids and thicker croissant-like scrolls. All filaments are helical in nature, with equal numbers of left- and right-handed filaments in each batch[2].

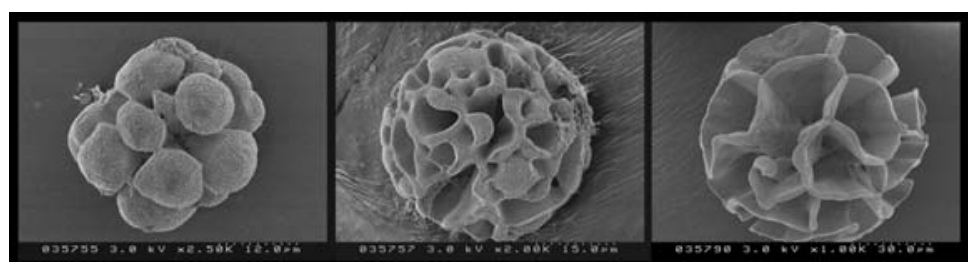

Fig. 2. FESEM images showing a growth sequence of floral spherulites.

These aggregates vary considerably in their external morphology with growth time, $\mathrm{pH}$ and concentration of the parent solution. In excess barium salt $(0.5 \mathrm{M})$, globular aggregates, resembling raspberries, eventually forming complex 'floral spherulites' (see Fig2). (Gel grown aggregates of this kind proceed to develop filamentous braids, extruded from isolated sites on the perimeter of the sheets [2]. X-ray Computed Tomography (X-ray CT) reveals the complex $3 \mathrm{D}$ nature and variable $\mathrm{X}$-ray contrast of these aggregates in some detail (see Fig2 C). Cross-sections of an X-ray tomogram of a floral spherulite (see Fig2 A,B) shows regions with high contrast (due to enhanced electron density (blue)) in the core of the aggregate as well as along the ('Plateau border') junction lines of the interconnecting sheets. Those variations are due to variable $\mathrm{Ba}$ :Si ratios in the aggregate. Higher ratios of Ba:Si species (likely witherite and silica) lead to enhanced X-ray contrast, suggesting that the outermost 
regions - the most sheet-like domains - are richer in silica than inner parts of the floral spherulites.
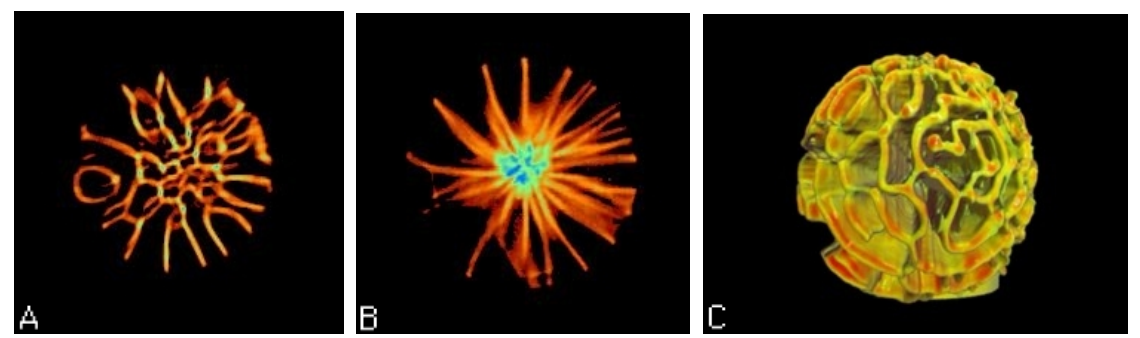

Fig. 3. Two X-ray CT cross-sections $(\mathbf{A}, \mathbf{B})$ of a floral spherulite and the corresponding $3 \mathrm{D}$ volume rendered image $(\mathbf{C})$.

\section{Microstructure of biomorphs}

In addition to the complex ultrastructres seen at the micron scale, a number of distinct structural features are found at the atomic $(\AA)$ and meso (nm) scales. Atomic scale ordering of barium and carbonates species is evident from Raman spectroscopy and X-ray (powder) diffraction. Both techniques confirm that $\mathrm{BaCO}_{3}$ is present as witherite crystals within the aggregates.

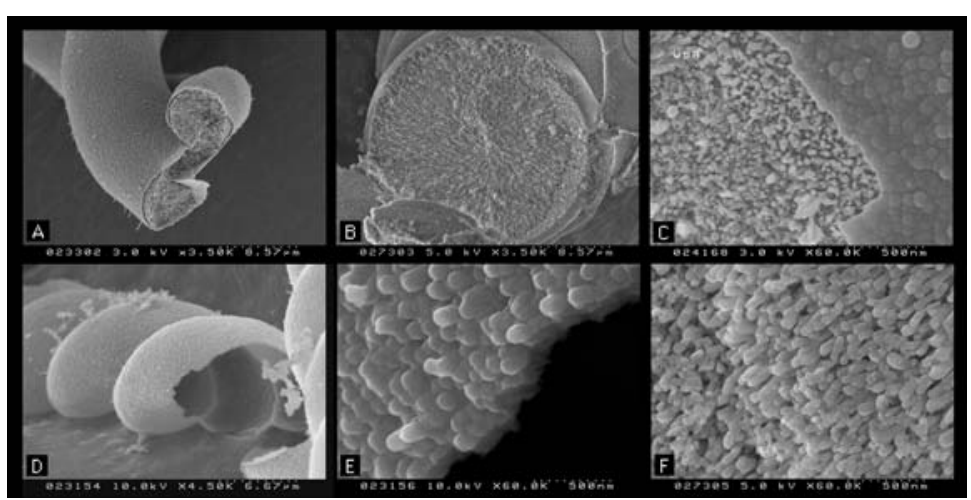

Fig. 4. FESEM images of slightly $\mathrm{NaOH}$ etched(A, $\mathbf{B}$ and $\mathbf{F})$, acid treated $(\mathbf{D}, \mathbf{E})$ and as-synthesised $(\mathbf{C})$ biomorphs.

The chemical nature of these biomorphs can be ascertained to some degree by selective dissolution in acidic or alkaline solutions. The former (usually done in a dilute solution of acetic acid) gently dissolves the carbonate material, leaving the silica intact. Immersion in dilute $\mathrm{NaOH}$ solution selectively dissolves the siliceous material, leaving the bare carbonate skeleton. Remarkably, neither treatment affects the ultrastructure of the biomorphs. Witherite dissolution leaves a hollow silica membrane, clearly visible in the optical microscope, whose micron-scale morphology is precisely that of the original biomorph (see Fig3 D and E). On the other hand, silica dissolution is also possible without disrupting the ultrastructural integrity of the material (see Fig3 B). 
These dissolution experiments suggest strongly that the biomorphs are dual composites, consisting of intimate intergrowths of witherite and amorphous silica. We cannot preclude the presence of other stoichiometries in the biomorphs, though these are less dominant than silica and barium carbonate. For example, more complex metal silicates may also exist in the undisturbed biomorphs [8]. The finer structure of the biomorphs is remarkable. Close inspection of the silica membrane shows that it is composed of a quasi-monodisperse distribution smaller silica spheres, 40nm in diameter. Selective removal of the silica reveals strikingly ordered arrangements of witherite material. Field emission scanning electron microscopy (FESEM) images show that the biomorphs are composed of myriad nanoscale witherite crystallites with a rod-like aspect. The globules are rather uniform, slightly elongated and about 50nm in dimension (see Fig3 D and F), though occasional extended stalk-like rods are seen. These aggregates are seen in all biomorphs, irrespective of their (larger-scale) morphology. Note that these forms are imaged after silica removal and alteration of the original carbonate material may occur during the dissolution process. Some ripening of the witherite crystallites may occur during silica dissolution, leading to monocrystalline particles, though the original nanorods may be polycrystalline.

The most striking aspect of FESEM images of the bare carbonate material is the orientational ordering between the nanorods within the biomorphs (see Fig3 A, B, E, F). Cross-sections through the sheets reveal bilateral symmetry, with incremental tilt of the rods from the mid-layer of the sheets towards both top and bottom faces.

Orientational order is also evident in optical micrographs of untreated sheets

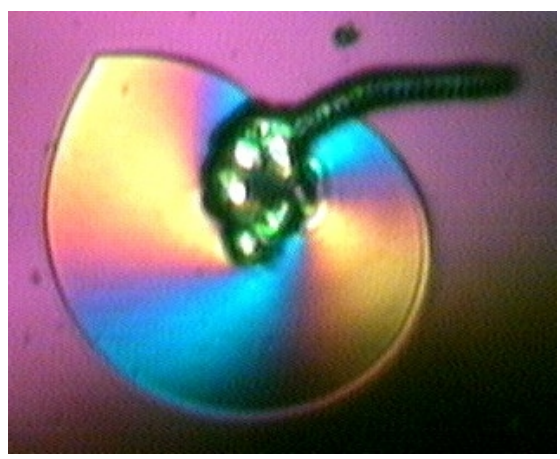

Fig. 5. Optical micrograph of a sheet viewed under crossed polarisers.

containing both silica and witherite. The optical textures consist of continuous radial variation of the interference colour between crossed polarisers with a sensitive tint plate inserted, and Maltese cross extinction patterns in the sheets, usually centred at the original 'raspberry' clusters from which the sheets grow radially outward (see Fig3). The distribution of interference colours in the sheets is consistent with radial orientation of the optically fast axis of witherite 
crystals outward from the central origin. (The fast axis of witherite is its crystallographic c axis, which is the pseudohexagonal axis and long direction of the prismatic witherite crystals.) Hence, the interference colours imply a wellordered but continuously varying orientation field for the witherite nanorods. Preliminary X-ray diffraction measurements support the finding of orientation ordering; selective streaking of some diffraction peaks has been seen, leading to novel diffraction patterns, intermediate to those of uncorrelated powders and single crystals [2].

FESEM images of intact silica-witherite biomorphs do not show the witherite nanorods clearly, as these are embedded within the siliceous globules (see Fig3C). The silica species are not likely to be later accretions to the condensed witherite material. Freshly harvested biomorphs (collected as soon as aggregates are detected in the optical microscope) reveal similar siliceous material to mature biomorphs, collected hours later. Thus, growth of the witherite and silica species occurs at about the same time. In addition, intergrowth of both species apprently occurs at virtually all length scales beyond the nm scale.

\section{Analogy with liquid crystals}

The presence of orientational ordering of witherite nanorods, in the absence of any translational order between adjacent rods, is suggestive of liquid crystals. Indeed, the analogy with chiral liquid crystals is irresistible. Consider the ordering within sheets, both within the "plane" of the sheets (see Fig3) and within a cross-section through the sheets (see Fig??). The tubular rims in the sheets are more highly curved than other sheet domains, and the twist between adjacent nanorods in these rims and braids that are extruded from the rims appear to be larger than in the sheets, as the nanorods are approximately the same size in all morphologies. A continuous rotation or tilt of the crystallite orientation is evident in both planes. This is reminiscent of the double-twist arrangement of chiral molecular species in some chiral mesophases, such as the twisted grain boundary (TGB) mesophase REF. In the biomorphs, the witherite-silica nanorods play the part of chiral pseudo-molecules. In contrast to the extended bulk TGB mesophase, the sheets are of finite extent, likely terminated once the local concentration of precipitating species drops below critical values.

A curious feature of all biomorphs is their apparent two-dimensional nature, despite the inherent three-dimensionality of the precursor ionic species in the crystallising medium. Clearly the outer regions of the floral spherulites and the extended sheets are surface-like. The intrinsic geometry of the braids is more difficult to isolate unequivocally, though these too appear to consist of laminated curved surfaces. For example, FESEM samples occasionally reveal 

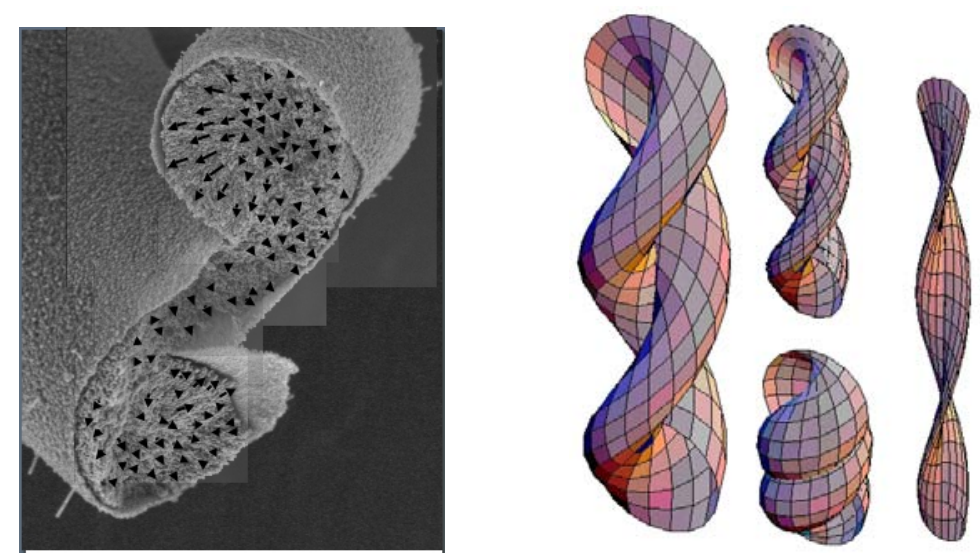

Fig. 6. Left: a composite FESEM image with added arrows showing the particle direction. Right: computer-generated twisted spheres.

a single curved layer dislodged from an adjacent braid.

\section{Global morphology from local constraints or local twist from global morphology}

We can extend the loose analogy to chiral liquid crystals, in order to explore whether the complex morphologies of the aggregates is a global geometry induced by local twist constraints on adjacent nanorods. There are some, but few, indications that local twist constraint can act in complex, particularly macromolecular, systems. Cholesteric mesophases are formed under the action of a local twist constraint, though this is a single twist (along only one of the three orthogonal rod axes) [10], rather than a double twist observed here. Cholesterics have been observed in collagen and chitin systems $[11,12]$, in addition to the simpler synthetic liquid crystalline molecular species. More recently, raft-like assemblies of polyelectrolyte molecules have been reported in the presence of added salts [5]. In addition, twisted packings are well known in protein structures, due to steric dense packing of macromolecules with welldefined chiral grooves, similar to close packing of threaded screws [13]. All of these examples require chiral building blocks. Our studies suggest that the nanorods consist of silica-decorated faces of the pseudo-hexagonal twins of orthorhombic witherite crystallites. None of the external faces of those witherite twins exhibit chirality. It is possible that the adsorbed silica on the faces of the witherite rods leads to an effective chirality. We are currently conducting preliminary molecular simulation experiments in order to investigate chiral silicate-carbonate adsorption. Even in the absence of a chiral charge distribution on the rods, the presence of polyanions in the condensing species may result in a preferred relative twist between the nanorods [2]. Charged rods have been suggested to condense with a fixed inclined angle between them 
under certain conditions [9].

An alternative explanation for the striking orientational ordering between nanorods can be drawn, converse to the previous one. Assume that the local twisted structure is the result of crystallisation of witherite rod-shaped crystallites within silica-rich curved films, whose shape is induced by external, global factors. Such factors have not been identified but they cannot be ruled out a priori. Flat silica membranes, consisting of aggregated silica (nm) spheres, have been reported for alkaline silica solutions in the presence of cationic surfactants, that screen the anionic silica spheres, allowing condensation [14]. Presumably, adjustment of the cationic species (Ba2+ in our case) allows the silica sphere coordination number to change, thereby adjusting the membrane curvature.

In that case, the observation of orientationally ordered witherite crystallites may be of incidental importance to the exotic curved morphologies seen in the biomorphs. Indeed, the crystallites may be due to recrystallisation of witherite after removal of silica species in alkaline solution. The presence of twist between adjacent crystallites is then solely due to the overall curvature of the sheets: the nanorod packing is that expected for small rods immersed in a curved film, assuming (for example) rods are aligned along growth directions of the aggregates. Indeed, the nanorods appear to be packed normal to the time-varying growth front of the biomorph, implying that the relative twist is a secondary effect of the growth habit. It is noteworthy that the floral spherulites display a sheet-like aspect in the presence of enhanced silica content at their outermost regions, while the inner, less surface-like, core apparently contains less silica. That too suggests that the silica plays a major role in structuring these biomorphs.

Biomorph geometries often show striking resemblances to standard surface forms found in texts on differential geometry. Both the sheets and trumpetbells are typically hyperbolic, indeed they mimic surfaces of constant negative Gaussian curvature ('pseudospheres'). Such a geometry is expected assuming a constant silica coordination number, exceeding six, in the silica membrane. In contrast, the thinner braids are very similar to 'twisted spheres', surfaces of variable (though mainly positive) Gaussian curvature, that are less readily explained by a simple sphere-packing argument. However, we can use those surfaces to construct detailed models for the nanorod assemblies in terms of the orientational field induced by foliations of parallel leaves. For example, an explicit model for the thinner braid structures consists of a stack of leaves, each parallel to an initial "seed" twisted surface. Two simpler possible packing modes of the witherite crystallites (the nanorod cores) can be imagined: one with the c-axis of the orthorhombic structure (coincident with the extended axis of the rods) aligned normal to the leaves, one with that axis parallel. 


\section{Evaluation of local/global scenarios}

Unfortunately, we still do not have sufficient evidence to distinguish between the two possible explanations advanced in the previous sections: we cannot decide whether the exotic curvilinear surface geometries of the raspberry, sheet and braid biomorphs are product of a local packing condition acting between nanorods or indeed whether the local packing is a by-product of the global surface-like geometries, whose forms are determined by other factors. We require more data and understanding of the system at theoretical and experimental levels.

Theoretical understanding of the self-assembly process resulting in biomorphs requires a physical model that can explain (i) the twist constraint leading to the sheet morphology and induction of the morphologies from that local constraint, or (ii) the sheet morphology (from which it seems to us that the twist constraint may follow readily) or (iii) a combination of both processes occurring simultaneously. We are exploring the structural evolution and dependence on added species in detail in order to elucidate this further. We can however already assert that the long-held distinction between the inorganic and organic realms, between solids and liquid crystals and the synthetic and biological worlds is overly simplistic.

\section{References}

[1] J. M. García-Ruiz, S. T. Hyde, A. M. Carnerup, A. G. Christy, M. J. van Kranendonk, and N. J. Welham. Self-assembled silica-carbonate structures and the detection of ancient microfossils. Science, 302:1194-1197, 14 November 2003 .

[2] J. M. García-Ruiz, A. Carnerup, A. G. Christy, N. J. Welham, and S. T Hyde. Morphology: an ambigous indicator of biogenicity. Astrobiology, 2(3):353-369, 2002 .

[3] J-C. P. Gabriel and P. Davidson. New trends in colloidal liquid crystals based on mineral moieties. Advanced Materials, 12:9-20, 2000.

[4] J-C. P. Gabriel, F. Camerel, B. J. Lemaire, H. Desvaux, P. Davidson, and P. Batail. Swollen liquid-crystalline lamellar phase based on extended solid-like sheets. Nature, 413:504-508, 2001.

[5] G. C. L. Wong, A. Lin, J. X. tang, Y. Li, P. A. janmey, and C. R. Safinya. Lamellar phase of stacked two-dimensional rafts of actin filaments. Physical Review letters, 91(1):018103-1 - 018103-4, July 2003.

[6] J. M. García-Ruiz. Carbonate precipitation into alkaline silica-rich environments. Geology, 26(9):843-846, 1998. 
[7] J. M. García-Ruiz. Carbonate sedimentation and diagenesis in the evolving Precambrian world, chapter Geochemical scenarios for the precipitation of biomimetic inorganic carbonates, pages 75-89. SEPM (Society for Sedimentary Geology) Special Publication, 67 edition, 2000.

[8] J. M. García-Ruiz. On the formation of induced morphology crystal aggregates. Journal of Crystal Growth, 73:251-262, 1985.

[9] R. Bruinsma. Liquid crystals of polyelectrolyte networks. Physical Review E, 63:016705, 2001.

[10] E. Grelet and S. Fraden. What is the origin of chirality in the cholesteric phase of virus suspensions? Physical Review Letters, 90:198302, 2003.

[11] M. M. Giraud-Guille. Liquid crystallinity in condensed type i collagen solutions: a clue to the packing of collagen in extracellular matrices. Journal of molecular biology, 224:861-873, 1992.

[12] S. B. Murray and A. C. Neville. The role of ph, temperature and nucleation in the formation of cholesteric liquid crystal spherulites from chitin and chitosan. International journal of biological macromolecules, 22(2):137-144, 1998.

[13] C. Brändén and J. Tooze. Intro to protein structure. Garland Publ Inc, 1991.

[14] Ralph K. Iler. The Chemistry of Silica : Solubility, Polymerization, Colloid and Surface Properties and Biochemistry of Silica. John Wiley and Sons Inc, 1979. 